\title{
La interacción entre padres e hijos y su relación con los problemas de conducta externalizante
}

\author{
Antonio Felix Raya Trenas, María José Pino Osuna y Javier Herruzo Cabrera \\ Universidad de Córdoba
}

ResUmen

El presente estudio se propone analizar la posible relación existente entre los problemas de conducta externalizante en los niños medidos por sus padres a través del BASC (Sistema de Evaluación de la Conducta de Niños y Adolescentes) y el estilo de crianza según el PCRI (Cuestionario de Crianza Parental) compuesto por siete factores: apoyo, satisfacción con la crianza, compromiso, comunicación, disciplina, autonomía y distribución de rol, en una muestra de 278 niños (152 niños y 126 niñas) entre 3 y 14 años. Los resultados muestran la existencia de una relación significativa entre los problemas de conducta externalizante en los niños y la mayoría de los factores del estilo de crianza parental. Además se establece un modelo capaz de predecir el 30,7\% de la varianza con respecto a los problemas de conducta externalizante en los niños, compuesto por la disciplina de ambos progenitores, la comunicación y la distribución de roles de los padres y el apoyo de las madres. Finalmente se discute la utilidad de estos resultados para el planteamiento de estrategias de intervención en el ámbito familiar basadas en el estilo disciplinario.

Palabras clave: problemas de conducta externalizante, interacción padres-hijos, estilo parental, disciplina

\section{ABSTRACT}

This current study proposes to analyse the possible relationship which exists between externalizing problem behaviour in children reported by parents through the BASC (Behaviour Assessment System for (hildren), and the parenting style according to the PCRI (Parent-Child Relationship Inventory) composed of seven factors such as support, satisfaction with parenting, involvement, communication, limit setting, autonomy granting, and role orientation, in a sample of 278 children ( 152 male \& 126 female) between 3 and 14 years old. The results show the existence of a significant relationship between externalizing problem behaviour in children and the majority of the parenting factors. Furthermore, a model is established which is able to predict $30,7 \%$ of the variance with respect to externalizing problem behaviour in children, made up by limit setting from both parents, communication and role orientation from the fathers, and support from the mothers. Finally, the utility of results to raise intervention strategies within the family based on limit setting is discussed.

Keywords: Externalizing problem behaviour, parent-child interaction, parenting style, limit setting 
Los problemas de conducta externalizante se caracterizan por conductas perturbadoras como la agresividad, la hiperactividad u otro tipo de comportamientos deshonestos como la transgresión de las normas sociales o los actos delictivos. Coinciden en buena medida con lo que Achenbach y Edelbrock (1978) denominaron conducta "insuficientemente controlada", siendo una característica clara la naturaleza perturbadora de la conducta del niño, que llama la atención de profesores, psicólogos y otros profesionales porque alteran las actividades de sus compañeros y de los adultos, son menos obedientes y presentan reacciones más problemáticas que los demás (Reynolds \& Kamphaus, 2004).

En el DSM-IV-TR (American Psychological Association [APA], 2002), estos problemas quedan recogidos dentro de la categoría de Trastornos por Déficit de Atención y Comportamiento Perturbador, donde podemos encontrar diagnósticos como el Trastorno Disocial, el Trastorno Negativista Desafiante y el Trastorno por Déficit de Atención e Hiperactividad. Estos trastornos presentan prevalencias que oscilan entre el 2 y el $16 \%$ en función del sexo o la edad de los chicos, aunque las conductas específicas que generan problemas de convivencia presentan una prevalencia estimada mucho más alta, sin que esto suponga la existencia de un diagnóstico (Schroeder \& Gordon, 1991).

Los problemas de conducta externalizante suelen empeorar con la edad, exacerbados por factores tales como el fracaso académico, el rechazo de los iguales o un estilo educativo ineficaz de los padres que carecen de habilidades para el gobierno de la familia. (Patter- son, 2002). Estudios recientes han calificado este modelo parental como disciplina negativa o disfuncional, que actúa como mediador entre la personalidad de padres e hijos y los problemas de conducta (Prinzie, Onghena, \& Hellinckx, 2006).

En referencia al modelo parental, el más conocido sin duda es el planteado por Baumrind (1967), reelaborado por Maccoby y Martin (1983). Este modelo planteaba la existencia de cuatro estilos parentales denominados autoritativo, autoritario, negligente e indulgente, que propiciaban en mayor o menor medida la aparición de problemas de conducta externalizante en los hijos. Basándose en este modelo, estudios recientes concluyeron que el estilo autoritativo era el que mejor prevenía estos problemas de conducta en los hijos, mientras que los estilos negligente e indulgente eran los que presentaban una relación más directa con estos problemas (López-Soler, Puerto, López-Pina, \& Prieto, 2009; McKinney \& Renk, 2011; Steinberg, Blatt-Eisengart, \& Cauffman, 2006; Villar, Luengo, Gómez, \& Romero, 2003).

La distinción que posteriormente establecieron Darling y Steinberg (1993) entre estilos parentales y prácticas de crianza ha marcado el devenir del estudio de la dinámica familiar en los últimos años. Análisis factoriales basados en estas prácticas de crianza han dado lugar a una serie de constructos referentes al comportamiento de los padres en la interacción diaria con los hijos. Uno de estos constructos denominado disciplina, consistencia o control conductual, ha sido contemplado por numerosos estudios que coinciden en indicar que unas pautas disciplinarias razonadas y consist- 
entes están relacionadas con una menor problemática en los hijos (Aunola \& Nurmi, 2005; Elgar, Waschbusch, Dadds, \& Sigvaldasson, 2007; Knutson, DeGarmo, \& Reid, 2004; McCoy, Frick, Money, \& Ellis, 1999; Oliva, Parra, Sánchez-Queija, \& López, 2007; Pfiffner, McBurnett, Rathouz, \& Judice, 2005; Raya, Pino, \& Herruzo, 2011; Tur, Mestre, \& Del Barrio, 2004). Uno de los principales requisitos para lograr establecer unas pautas disciplinarias consistentes y razonadas es la existencia de una relación adecuada entre padres e hijos. En esta línea, numerosos investigadores relacionan los problemas de conducta externalizante con aspectos como la mala comunicación (Krinsley \& Bry, 1992; Villar et al., 2003) o la falta de implicación y compromiso con la crianza de los hijos (Finkenauer, Engels, \& Baumeister, 2005; Kimonis et al., 2006; McCoy et al., 1999).

Otro aspecto frecuentemente estudiado es el grado de autonomía que, según los trabajos de Bynum y Kotchick (2006), Reitz, Dekovic y Meijer (2006) o Tur et al. (2004), fomenta la responsabilidad y está relacionada con una menor problemática en el niño. No obstante, otros trabajos como los de Beyers, Bates, Pettit y Dodge (2003), Raya, Pino y Herruzo (2009) o Vazsonyi (2004) concluían que un elevado grado de autonomía y falta de supervisión podría identificarse con una actitud negligente por parte de los padres, que daría lugar a una situación de riesgo.

Por otro lado, existen otras variables que reflejan el grado de apoyo percibido en la crianza y que han sido relacionadas con los problemas de conducta externalizante, como la pobreza crónica (Pachter, Auinger, Palmer, \& Weitzman,
2006) o la situación de desempleo (Knutson et al., 2004). También la mala calidad de las redes sociales ha sido indicada como una fuente de estrés e insatisfacción en la crianza (Vandewater \& Lansford, 2005).

Estudios anteriores llevados a cabo con población española han mostrado que variables como la implicación, la comunicación, el apoyo percibido o la disciplina guardan una estrecha relación con determinados problemas de comportamiento en los hijos como la hiperactividad y la impulsividad (Raya, Herruzo, \& Pino, 2008; Robledo Román, García Sánchez, \& Díez González, 2009) o la conducta agresiva (Raya et al., 2009; Samper, Aparici, \& Mestre, 2006). En definitiva, esta relación entre determinados aspectos del estilo de crianza y ciertas manifestaciones conductuales inadecuadas, nos permite plantear que, llevando a cabo una medición de determinadas características del estilo de crianza de los padres, se podría predecir en cierta medida la presencia de otros problemas de adaptación en los hijos como es el caso de los problemas de conducta externalizante. En este sentido, este estudio se propone describir la relación existente entre este tipo de problemas en una muestra de escolares y el estilo de crianza de sus padres y madres. Además, se pretende elaborar un modelo predictor de los problemas de conducta externalizante en los hijos compuesto por distintos factores del estilo de crianza de los padres y madres.

\section{Método}

\section{Participantes}

Para la selección de un amplio número de 
participantes de estatus socioeconómico medio, se recurrió a tres centros de Educación Infantil, Primaria y Secundaria, situados en dos localidades de la provincia de Córdoba y una de la provincia de Jaén. Así, se obtuvo información de 278 chicos y chicas con sus respectivos padres y madres.

La edad media de los padres era de 40.11 $(D T=5,503)$, con un rango entre 28 y 70 años, mientras que la edad media de las madres era de 38,13 ( $D T=4,977)$, situándose el rango en este caso entre 26 y 56 años.

Por su parte, la muestra de hijos estaba compuesta por 152 niños y 126 niñas, con una edad media de 8,27 (DT=3,187), con un mínimo de 3 años, un máximo de 14.

\section{Instrumentos}

Para la recogida de la información se han empleado los siguientes instrumentos:

- Sistema de evaluación de la conducta de niños y adolescentes BASC (Reynold \& Kamphaus, 2004): es una adaptación al castellano del original en lengua inglesa Behavior Assessment System for Children.

Evalúa una amplia gama de dimensiones patológicas y adaptativas, mediante distintas fuentes de información y distintos métodos. Para este estudio se ha usado el cuestionario para padres.

Este cuestionario se divide en tres niveles según la edad $(3-6,6-12,12-18)$ y presenta un índice de consistencia interna entre 0,70 y 0,90 , según las escalas. Respecto a la correlación test-retest (intervalo de tres meses), los valores son $0,85,0,88$ y 0,70 para los tres niveles del cuestionario.
De las distintas escalas que contempla el instrumento, para este estudio se ha usado la dimensión global denominada Exteriorizar problemas, que deriva a su vez de otras tres escalas referentes a la conducta perturbadora como son la agresividad, la hiperactividad y los problemas de conducta. Esta escala presenta unos índices de consistencia interna medidos mediante el coeficiente a de Cronbach entre 0,86 y 0,88 .

Las puntuaciones obtenidas en cualquiera de las escalas son transformadas en puntuaciones $T$, que señalan la distancia de una puntuación con respecto a la media del grupo normativo y permiten establecer comparaciones entre sujetos de diferentes edades. Estas puntuaciones T pueden oscilar entre 0 y 100 y presentan una media de 50 y una desviación típica de 10. En base a estas puntuaciones T se establecen distintos niveles, de modo que puntuaciones por debajo de 30 se consideran muy bajas, por debajo de 40 bajas, entre 40 y 60 nivel medio o normal, por encima de 60 en riesgo y por encima de 70 clínicamente significativo.

- Cuestionario de Crianza Parental (PCRI-M), de Roa y Del Barrio (2001), adaptado del Parent-Child Relationship Inventory (Gerard, 1994): este cuestionario mide las actitudes paternas y maternas hacia la crianza de sus hijos.

Está compuesto por 78 items con cuatro opciones de respuesta, que se agrupan en siete escalas:

- Apoyo: nivel de apoyo social y emocional que una madre o padre está recibiendo.

- Satisfacción con la crianza: satisfacción que obtiene una madre o padre por el hecho de serlo. 
- Compromiso: grado de interacción y conocimiento que los padres tienen de su hijo.

- Comunicación: percepción que los padres tiene de la efectividad de la comunicación con su hijo.

- Disciplina: nivel de exigencia de cumplimiento de las normas impuesto por los padres.

- Autonomía: habilidad de los padres para proporcionar independencia al hijo.

- Distribución de rol: actitudes de los padres acerca del papel que desempeña el género en la crianza.

En general, una alta puntuación en cualquiera de las escalas indica una buena actitud hacia la crianza (estilo democrático), mientras que puntuaciones bajas son signo de un estilo de crianza poco efectivo.

La consistencia interna del instrumento para esta muestra, obtenida mediante el coeficiente alfa de Cronbach, oscila entre 0,68 de la escala de Apoyo y 0,78 de la escala de Satisfacción con la crianza. Además, podemos decir que el cuestionario presenta una buena validez de constructo, dadas las correlaciones obtenidas entre las distintas escalas del cuestionario, sobre todo en las escalas más importantes de la crianza como son CompromisoSatisfacción con la crianza $(0,51)$, CompromisoComunicación $(0,64)$, Disciplina-Apoyo $(0,42)$, Disciplina-Autonomía $(0,44)$ y Satisfacción con la crianza-Disciplina $(0,37)$.

\section{Procedimiento}

Para contactar con las familias participantes recurrimos a los centros donde estaban esco- larizados sus hijos. El primer paso fue obtener permiso de los equipos directivos y consejos escolares de los distintos centros para participar en el estudio. Para informar a las familias por escrito y hacerles llegar el instrumento contamos con la colaboración de los tutores de los distintos cursos, que se encargaron tanto de distribuir como de recoger de los cuestionarios. Las familias, que accedieron a colaborar de manera voluntaria, cumplimentaron el BASC en su forma $P$ (padres) y en sus distintas versiones según la edad de los chicos y el PCRI tanto por parte del padre como de la madre.

Tras recoger los cuestionarios se pasó a la tabulación de los datos, transformación de las puntuaciones del BASC en puntuaciones $\mathrm{T}$ como se indica en la descripción del instrumento y realización de los análisis con el paquete estadístico SPSS.

\section{Análisis de datos}

El estudio se ha realizado con un diseño ex post facto prospectivo en el que se ha realizado un análisis de regresión múltiple para obtener los factores predictores de la agresividad. Por lo tanto, las variables empleadas en el estudio fueron las siguientes: como variable dependiente (VD) se ha usado la puntuación T en exteriorizar problemas y como variables predictoras los factores del estilo de crianza tanto del padre como de la madre medidos con el PCRI-M, que son: apoyo, satisfacción con la crianza, compromiso, comunicación, disciplina, autonomía y distribución de rol.

Previamente al análisis de regresión múltiple se han descrito las puntuaciones obtenidas en los distintos instrumentos y se ha calculado 
el coeficiente de correlación de Pearson entre la variable exteriorizar problemas y los factores del estilo de crianza de padres y madres.

\section{Resultados}

Para la variable agresividad, se ha obtenido una puntuación T media de 48,35 ( $D T=9,616)$, que resultó ser un poco más baja que la media establecida por el instrumento $(T=50)$. Las puntuaciones obtenidas por los participantes se sitúan en un rango entre 28 y 85 .

Respecto a la clasificación de los sujetos en función de los niveles establecidos por el BASC, la Figura 1 muestra el porcentaje de sujetos que han sido clasificados como muy adapta$\operatorname{dos}(\mathrm{T}<30)$, adaptados $(30<\mathrm{T}<40)$, dentro de un rango normal $(40<T<60)$, en riesgo ( $T>$ 60) y clínicamente significativos ( $T>70$ ).

Posteriormente, para identificar aquellas variables relacionadas con los problemas de conducta externalizante, se calculó el coeficiente de correlación de Pearson entre la variable exteriorizar problemas y todos los factores del PCRI de padres y madres, cuyas puntuaciones medias se muestran en la Tabla 1. Todas las variables han correlacionado de manera inversa con la variable exteriorizar problemas. Además, todas excepto la distribución de roles

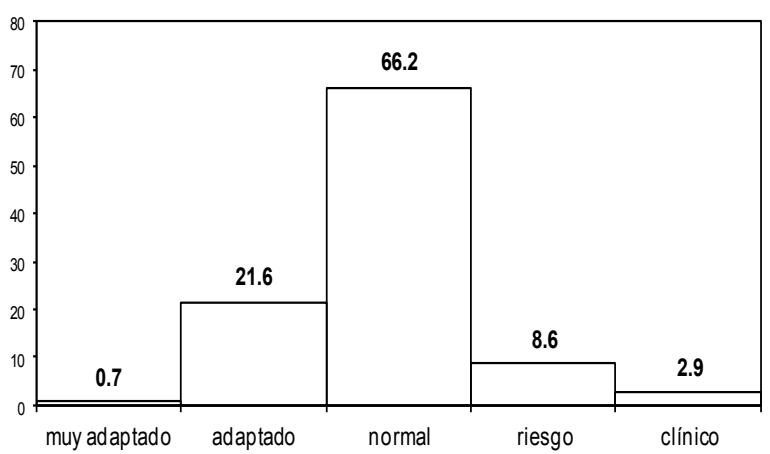

Figura 1. Clasificación de los chicos según su puntuación en exteriorizar problemas. de ambos progenitores lo han hecho de manera significativa, luego altas puntuaciones en estas variables están relacionadas con bajas puntuaciones en exteriorizar problemas.

Finalmente, para estudiar el carácter predictor de los factores del PCRI respecto a la agresividad, se aplicó el análisis de regresión múltiple mediante el que se pretende obtener un modelo capaz de predecir la posibilidad de presentar una alta puntuación en problemas de conducta externalizante. Las cinco variables que componían el modelo con mayor capacidad predictora eran la disciplina de la madre, la disciplina del padre, la comunicación del padre, la distribución de rol del padre y el apoyo de la madre, como muestra la Tabla 2.

En el quinto paso se obtuvo una $R^{2}$ de 0,307 , por lo que se puede decir que las variables disciplina de la madre, disciplina del padre, comunicación del padre, distribución de rol del padre y apoyo de la madre predicen el 30,7\% de la variabilidad respecto a los problemas de conducta externalizante. Para todas estas variables se han obtenido coeficientes Beta negativos excepto para la distribución de rol del padre, por lo que esta variable es predictiva de una puntuación alta en problemas de conducta externalizante cuando presenta puntuaciones también altas. Para el resto de las variables ocurre lo contrario.

Todas las variables incluidas hacen una aportación interesante al modelo, que mejora ostensiblemente en cada paso. Por su parte, los estadísticos de colinealidad que nos informan sobre la independencia de cada factor respecto a los demás, ofrecen unos resultados bastante buenos, teniendo en cuenta que el 
Tabla 1

Puntuación media para cada factor del PCRI y coeficientes de correlación de Pearson entre los factores del PCRI de padres y madres y la variable exteriorizar problemas

\begin{tabular}{|c|c|c|c|c|c|c|c|}
\hline PCRI padre & $M$ & $D T$ & $\begin{array}{c}\text { Correlación con } \\
\text { Exteriorizar problemas }\end{array}$ & PCRI madre & $M$ & $D T$ & $\begin{array}{c}\text { Correlación con } \\
\text { Exteriorizar problemas }\end{array}$ \\
\hline$\overline{\text { Apoyo }}$ & 25,31 & 3,627 & $-0,273^{* *}$ & Apoyo & 23,83 & 4,096 & $-0,342 * *$ \\
\hline Satisfacción & 34,82 & 4,031 & $-0,261^{* *}$ & Satisfacción & 34,80 & 4,185 & $-0,265^{* *}$ \\
\hline Compromiso & 43,66 & 4,366 & $-0,236^{* *}$ & Compromiso & 44,99 & 4,031 & $-0,310^{* *}$ \\
\hline Comunicación & 28,65 & 3,353 & $-0,228 * *$ & Comunicación & 29,72 & 3,611 & $-0,245^{* *}$ \\
\hline Disciplina & 32,07 & 4,503 & $-0,404^{* *}$ & Disciplina & 31,70 & 4,802 & $-0,445^{* *}$ \\
\hline Autonomía & 24,91 & 2,950 & $-0,190^{* *}$ & Autonomía & 25,22 & 3,416 & $-0,146^{*}$ \\
\hline Distrib. rol & 27,56 & 4,146 & $-0,070$ & Distrib. rol & 28,27 & 4,016 & $-0,106$ \\
\hline
\end{tabular}

$* p<0,05 * * p<0,01$

valor de tolerancia está más próximo a 1 que a 0 y que el factor de inflación de la varianza (FIV) no se aleja demasiado de 1.

\section{Discusión y conclusiones}

Los datos descriptivos mostrados en nuestro estudio son un buen indicativo de la representatividad de la muestra con la que hemos trabajado. Así, la muestra presenta una puntuación media en exteriorizar problemas bastante cercana a la media normativa, mientras que la distribución en base a los criterios de clasificación del BASC presenta una alta concentración de sujetos en el rango "normal", reduciéndose los sujetos clínicamente significativos a un $2,9 \%$, lo que resulta bastante representativo de la población general, donde las tasas de prevalencia para el Trastorno Disocial oscilan entre el 2 y el 16\% (APA, 2002).

Este estudio se proponía analizar la relación existente entre el estilo de crianza de ambos progenitores y los problemas de conducta externalizante en los hijos. En la introducción se apuntaba cierta relación que numerosos autores habían descrito entre determinados aspectos del estilo de crianza de los padres y los problemas de conducta externalizante en los hijos, de manera que podrían llegar a establecerse determinados modelos familiares directamente relacionados con los mismos. En este sentido, asumiendo ciertas limitaciones como el tratarse de un estudio transversal o el uso de una única fuente de información (los padres), los resultados obtenidos reflejan una elevada relación entre determinadas características del estilo de crianza y los problemas conducta externalizante, de manera que una combinación de puntuaciones altas en distribución de rol del padre junto con puntuaciones bajas en apoyo percibido por la madre, comunicación del padre y disciplina de ambos progenitores, influyen significativamente en el incremento de la probabilidad de que se dé una puntuación elevada en problemas de conducta externalizante según el BASC. Mediante estas variables hemos podido explicar más del $30 \%$ de la varianza respecto a estos problemas, lo que consideramos una cifra bastante importante si tenemos en cuenta la naturaleza multidimensional de los problemas de conducta externalizante, en cuya aparición y mantenimiento pueden influir aspectos tan diversos como la 
Tabla 2

Regresión múltiple en pasos sucesivos para la VD exteriorizar problemas

\begin{tabular}{|c|c|c|c|c|c|c|c|c|c|c|c|c|}
\hline \multirow[t]{2}{*}{ Variable } & \multirow[t]{2}{*}{$R$} & \multirow[t]{2}{*}{$R^{2}$} & \multirow{2}{*}{$\begin{array}{c}R^{2} \\
\text { corr. }\end{array}$} & \multirow[t]{2}{*}{$F$} & \multirow[t]{2}{*}{ sig. } & \multirow[t]{2}{*}{ B } & \multirow[t]{2}{*}{$t$} & \multirow[t]{2}{*}{ sig. } & \multicolumn{2}{|c|}{ Int. para B 95\% } & \multicolumn{2}{|c|}{ Colinealidad } \\
\hline & & & & & & & & & Inf. & Sup. & Tol. & FIV \\
\hline$\overline{\text { Paso } 1}$ & 0,445 & 0,198 & 0,195 & 68,16 & 0,000 & & & & & & & \\
\hline Constante & & & & & & 76,598 & 22,135 & 0,000 & 69,785 & 83,410 & & \\
\hline Disciplina madre & & & & & & $-0,891$ & $-8,256$ & 0,000 & $-1,104$ & $-0,679$ & 1,000 & 1,000 \\
\hline Paso 2 & 0,499 & 0,249 & 0,244 & 45,65 & 0,000 & & & & & & & \\
\hline Constante & & & & & & 86,650 & 21,244 & 0,000 & 78,620 & 94,680 & & \\
\hline Disciplina madre & & & & & & $-0,659$ & $-5,606$ & 0,000 & $-0,890$ & $-0,428$ & 0,792 & 1,262 \\
\hline Disciplina padre & & & & & & $-0,543$ & $-4,331$ & 0,000 & $-0,790$ & $-0,296$ & 0,792 & 1,262 \\
\hline Paso 3 & 0,531 & 0,282 & 0,274 & 35,83 & 0,000 & & & & & & & \\
\hline Constante & & & & & & 100,329 & 18,000 & 0,000 & 89,356 & 111,301 & & \\
\hline Disciplina madre & & & & & & $-0,613$ & $-5,292$ & 0,000 & $-0,842$ & $-0,385$ & 0,782 & 1,278 \\
\hline Disciplina padre & & & & & & $-0,549$ & $-4,472$ & 0,000 & $-0,791$ & $-0,308$ & 0,792 & 1,263 \\
\hline Comunicación padre & & & & & & $-0,521$ & $-3,521$ & 0,001 & $-0,812$ & $-0,230$ & 0,986 & 1,014 \\
\hline Paso 4 & 0,543 & 0,295 & 0,285 & 28,58 & 0,000 & & & & & & & \\
\hline Constante & & & & & & 96,655 & 16,776 & 0,000 & 85,313 & 107,998 & & \\
\hline Disciplina madre & & & & & & $-0,662$ & $-5,657$ & 0,000 & $-0,892$ & $-0,431$ & 0,757 & 1,322 \\
\hline Disciplina padre & & & & & & $-0,588$ & $-4,776$ & 0,000 & $-0,830$ & $-0,346$ & 0,777 & 1,287 \\
\hline Comunicación padre & & & & & & $-0,570$ & $-3,845$ & 0,000 & $-0,863$ & $-0,278$ & 0,965 & 1,037 \\
\hline Distribución padre & & & & & & 0,285 & 2,279 & 0,023 & 0,039 & 0,532 & 0,886 & 1,129 \\
\hline Paso 5 & 0,554 & 0,307 & 0,295 & 24,13 & 0,000 & & & & & & & \\
\hline Constante & & & & & & 97,766 & 17,018 & 0,000 & 86,456 & 109,076 & & \\
\hline Disciplina madre & & & & & & $-0,548$ & $-4,302$ & 0,000 & $-0,799$ & $-0,297$ & 0,630 & 1,588 \\
\hline Disciplina padre & & & & & & $-0,588$ & $-4,813$ & 0,000 & $-0,829$ & $-0,348$ & 0,777 & 1,287 \\
\hline Comunicación padre & & & & & & $-0,484$ & $-3,175$ & 0,002 & $-0,785$ & $-0,184$ & 0,900 & 1,111 \\
\hline Distribución padre & & & & & & 0,287 & 2,310 & 0,022 & 0,042 & 0,532 & 0,886 & 1,129 \\
\hline Apoyo madre & & & & & & $-0,303$ & $-2,180$ & 0,030 & $-0,577$ & $-0,029$ & 0,727 & 1,376 \\
\hline
\end{tabular}

familia, los iguales o los medios de comunicación.

En general, la mayoría los factores del estilo de crianza parental medidos muestran una fuerte relación inversa con los problemas de conducta externalizante. Además, aquellas variables que han resultado ser más importantes y por tanto más válidas para la composición de los modelos, confirman los resultados obtenidos por autores como Aunola y Nurmi (2005), Elgar et al. (2007), Knutson et al. (2004), McCoy et al. (1999), Pfiffner et al. (2005) o Tur et al. (2004). En este sentido, podemos decir que un bajo nivel de disciplina, entendida como establecimiento de unos límites claros, actúa como un excelente predictor de los problemas de conducta externalizante. Algo parecido ocurre con la comunicación con de los hijos, que también aparecía en los estudios revisados como predictor de unos mejores niveles de adaptación en los hijos. En cuanto al apoyo social y emocional percibido por las madres, hemos podido confirmar que, tal y como planteaban algunos autores como Vandewater y Lansford, (2005), un bajo grado de apoyo social está relacionado con una mayor problemática en los hijos y con un sentimiento de mayor insatisfacción con el rol parental. Sin embargo, la distribución de rol entendida como creencia en el reparto igualitario de las tareas en el hogar ha 
sido incluida en nuestro modelo como un componente negativo respecto a la adaptación de los hijos, aunque estos resultados adquieren cierta lógica cuando los encuadramos dentro de un modelo familiar de padres negligentes y poco comunicativos y escasos recursos de apoyo en las tareas de crianza. Otras variables como la autonomía, el compromiso o la satisfacción con la crianza, que no han formado parte del modelo de regresión múltiple, presentaban también una relación inversa y significativa con los problemas de conducta externalizante, dando buena cuenta de su carácter protector a pesar de no ser los predictores más importantes según nuestro modelo.

La información obtenida ha de ser analizada asumiendo la existencia de una relación bidireccional entre padres e hijos, considerando que ambos ejercen una influencia mutua en sus comportamientos y actitudes. Visto desde esta perspectiva interactiva entre padres e hijos, estos resultados tiene una gran aplicación tanto en la prevención como en la intervención con familias de niños problemáticos, pues puede orientar sobre algunos aspectos concretos de la práctica educativa diaria que pueden ser modificados, como enseñar a los padres a establecer unas normas claras y asegurar su cumplimiento o mejorar las redes de apoyo social y familiar. Estas pautas de prevención e intervención podrían verse enriquecidas con nuevos estudios que aporten información sobre determinadas variables familiares relacionadas con otros aspectos positivos del desarrollo como las habilidades adaptativas $u$ otros negativos como los problemas de conducta internalizante.

\section{Referencias}

Achenbach, T. M., \& Edelbrock, C. S. (1978). The classification of child psychopatology: A review and analysis of empirical efforts. Psychological Bulletin, 85, 1275-1301. doi:10.103/F0033-2909.85.6.1275

American Psychological Association. (2002). Manual diagnóstico y estadístico de los trastornos mentales (4. ${ }^{\text {a }}$ ed., texto rev.) ( $\mathrm{T}$. de Flores, J. Toro, J. Masna, J. Treserra, É. Masana, \& C. Udina, Trads.). Barcelona: Masson (Trabajo original publicado en 2000).

Aunola, K. \& Nurmi, J. E. (2005). The role of parenting styles in children's problem behavior. Child Development, 76, 1144-1159. doi:10.1111/j.1467-8624.2005.00840.x-i1

Baumrind, D. (1967). Child care practices anteceding three patterns of preschool behaviour. Genetic Psychology Monographs, 75, 43-88. doi:10.230/F1127295

Beyers, J. M., Bates, J. E., Pettit, G. S., \& Dodge, K. A. (2003). Neighbourhood structure, parenting processes, and the development of youths' externalizing behaviors: A multievel analysis. American Journal of Community Psychology, 31, 35-53. doi:10.1023/A:1023018502759

Bynum, M. S., \& Kotchick, B.A. (2006). MotherAdolescent Relationship Quality and Autonomy as Predictors of Psychosocial Adjustment Among African American Adolescents. Journal of Child and Family Studies, 15, 529-542. doi:10.1007/s10826-006-9035-z

Darling, N., \& Steinberg, L. (1993). Parenting style as context: An integrative model. Psychological Bulletin, 113, 487-496. doi:10.1037/0033-2909.113.3.487

Elgar, F. J., Waschbusch, D. A., Dadds, M. R., \& Sigvaldasson, N. (2007). Development and validation of a short form of the Alabama Parenting Questionnaire. Journal of Child and Family Studies, 16, 243-259. doi:10.1007/s10826-006-9082-5 
Finkenauer, C., Engels, R. C., \& Baumeister, R. F. (2005). Parenting behaviour and adolescent behavioural and emotional problems: The role of self-control. International Journal of Behavioral Development, 29, 58-69. doi:10.1080/01650250444000333

Gerard, A. (1994). Parent-Child Relationship Inventory: Manual. Los Angeles: Western Psychological Services.

Kimonis, E. R., Frick, P. J., Boris, N. W., Smyke, A. T., Cornell, A. H., Farrell, J. M., \& Zeanah, C. H. (2006). Callous-unemotional features, behavioral inhibition, and parenting: independent predictors of aggression in a highrisk preschool sample. Journal of Child and Family Studies, 15, 745-756. doi:10.1007/ s10826-006-9047-8

Knutson, J. F., DeGarmo, D. S., \& Reid, J. B. (2004). Social disadvantage and neglectful parenting as precursors to the development of antisocial and aggressive child behavior: Testing a theoretical model. $\mathrm{Ag}$ gressive Behavior, 30, 187-205. doi:10.1002/ ab.20016

López-Soler, C., Puerto, J. C., López-Pina, J. A., \& Prieto, M. (2009). Percepción de los estilos educativos parentales e inadaptación en menores pediátricos. Anales de Psicología, $25,70-77$.

Krinsley, K. E., \& Bry, B.H. (1992). Sequential analyses of adolescent, mother and father behaviors in distressed and non distressed families. Child \& Family Behavior Therapy, 13,45-62. doi:10.1300/J019v13n04_03

Maccoby, E. E., \& Martin, J. A. (1983). Socialization in the context of the family: Parentchild interaction. En P. H. Mussen (Ed.), Handbook of Child Psychology (Vol. 4, pp. 1-101). Nueva York: John Wiley \& Sons.

McCoy, M. G. Frick, P. J., Loney, B. R., \& Ellis, M. L. (1999). The potential mediating role of parenting practices in the development of conduct problems in a clinic-referred sample. Journal of Child and Family Studies, 8, 477-494. doi:10.1023/A:1021907905277
McKinney, C., \& Renk, K. (2011). A multivariate model of parent-adolescent relationship variables in early adolescence. Child Psychiatry and Human Development, 42(4), 44262. doi:10.1007/s10578-011-0228-3

Oliva, A., Parra, A., Sánchez-Queija, I., \& López, F. (2007). Estilos educativos materno y paterno: Evaluación y relación con el ajuste adolescente. Anales de Psicología, 23, 49-56.

Pachter, L. M., Auinger, P., Palmer, R., \& Weitzman, M. (2006). Do parenting and the home environment, maternal depression, neighbourhood, and chronic poverty affect child behavioral problems differently in different racial-ethnic groups? Pediatrics, 117, 1329-1338. doi:10.1542/peds.20051784

Patterson, G. R. (2002). The early developmental of coercitive family process. En J. B. Reid, G. R. Patterson, \& J. Snyder (Eds.), Antisocial behavior in children and adolescents: Developmental theories and models for intervention (pp. 25-44). Washington, DC: American Psychological Association. doi:10.1037/10468-002

Pfiffner, L. J., McBurnett, K., Rathouz, P. J., \& Judice S. (2005). Family correlates of oppositional and conduct disorders in children with attention deficit/hyperactivity disorder. Journal of Abnormal Child Psychology, 33, 551-563. doi:10.1007/s10802-0056737-4

Prinzie, P., Onghena, P., \& Hellinckx, W. (2006). A cohort-sequential multivariate latent growth curve analysis of normative $\mathrm{CBCL}$ aggressive and delinquent problem behavior: Associations with harsh discipline and gender. International Journal of Behavioral Development, 30, 444-459. doi:10.1177/0165025406071901

Raya, A. F., Herruzo, J., \& Pino, M. J. (2008). El estilo de crianza parental y su relación con la hiperactividad. Psicothema, 20, 691-696.

Raya, A. F., Pino, M. J., \& Herruzo, J. (2009). La agresividad en la infancia: el estilo de cri- 
anza parental como factor relacionado. European Journal of Education and Psychology, 2, 211-222. Recuperado de http://www. ejep.es/index.php/journal/article/view/33/ pdf_42

Raya, A. F., Pino, M. J., \& Herruzo, J. (2011). Family variables related to behavioral problems in childhood. Israel Journal of Psychiatry, 48(2), 117-122.

Reitz, E., Dekovic, M., \& Meijer, A. M. (2006). Relations between parenting and externalizing and internalizing problem behaviour in early adolescence: Child behaviour as moderator and predictor. Journal of Adolescence, 29, 419-436. doi:10.1016/j.adolescence.2005.08.003

Reynolds, C. R., \& Kamphaus, R. W. (2004). Sistema de evaluación de la conducta de niños $y$ adolescentes. Madrid: TEA Ediciones.

Roa, L., \& Del Barrio, V. (2001). Adaptación del cuestionario de crianza parental (PCRI-M) a población española. Revista Latinoamericana de Psicología, 33, 329-341.

Robledo Román, P., García Sánchez, J. N., \& Díez González, C. (2009). Relación entre la tipología de alumnos y factores psicológicos parentales en familias de personas con y sin dificultades de aprendizaje y TDAH. Análisis y Modificación de Conducta, 35, 1-18.

Samper, P., Aparici, G., \& Mestre, V. (2006). La agresividad auto y heteroevaluada: variables implicadas. Acción Psicológica, 2, 155168.

Schroeder, C. S., \& Gordon, B. N. (1991). Assessment and treatment of childhood problems: A clinician's guide. Nueva York: Guilford.

Steinberg, L., Blatt-Eisengart, I., \& Cauffman, E. (2006). Patterns of competence and adjustment among adolescents from authoritative, authoritarian, indulgent and neglectful homes: A replication in a sample of serious juvenile offenders. Journal of Research on Adolescence, 16, 47-58. doi:10.1111/ j.1532-7795.2006.00119.x
Tur, A., Mestre, V., \& Del Barrio, M. V. (2004). Los problemas de conducta exteriorizados e interiorizados en la adolescencia: relaciones con los hábitos de crianza y con el temperamento. Acción Psicológica, 36, 207-221.

Vandewater, E. A., \& Lansford, J. E. (2005). A family process model of problem behaviors in adolescents. Journal of Marriage and Family, 67, 100-109. doi:10.1111/j.00222445.2005.00008.x

Vazsonyi, A. T. (2004). Parent-Adolescent relations and problem behaviors: Hungary, the Netherlands, Switzerland, and the United States. Marriage and Family Review, 35, 161187.

Villar, P., Luengo, M. A., Gómez, J. A., \& Romero, E. (2003). Una propuesta de evaluación de variables familiares en la prevención de la conducta problema en la adolescencia. Psicothema, 15, 581-588. 
